\title{
Mixing of Particulate Solids ${ }^{\dagger}$
}

\author{
Karl Sommer \\ Technische Universität München* \\ Lehrstuhl für Maschinen- und Apparatekunde
}

\begin{abstract}
Solids mixing refers to the blending of particulate components differing in one of their properties. To compare mix and mixer the theoretical standard deviation $\sigma$ is defined as the mixing quality. To simulate the stochastic mixing process the Fokker-Planck-Equation is used. Similar simulations described in the literature up to now, in which the transport coefficient is determined from the throughput, are not generally valid. It could be shown with the modified method that the mixing results depend only on the relationship between the residence time and the fluctuation time of the feed material streams.

A model, based on a suggestion of Schulze, is useful to simulate deterministic silo mixers. The experiments confirm the results of the simulations.
\end{abstract}

\section{Introduction}

The mixing of solids is one of the oldest and most frequently performed of all chemical engineering operations. With increasing automation of engineering processes in all material transformation sectors, the application of scientific principles to such operations is of increasing importance $[1,2,3]$.

Solids mixing refers to the blending of at least one solids component with another, whereby the particulate components differ in at least one of their properties. The definition of mixing efficiency often differs depending on whether one is a manufacturer or customer of mixed materials and mixers. One should therefore specifically define "mixing efficiency" if this parameter is to be used to assess the quality of a mixed material. While solids mixing has been accomplished for centuries in batch operations, just as in other unit operations (e.g. stirred tank reactions, tubular reactors, combustion chambers, etc.), there is now a strong tendency to conduct solids mixing in a continuous fashion.

\section{Mixing efficiency}

The determination of mixing efficiency is a statistical problem and is coupled with the problem of sample collection. The goal of mixing is, of course, to distribute the various components as completely as possible throughout the material. When the component concentration at localised points (i.e. in collected samples) differs from the overall mix composition,

* Weihenstephan, 85350 Freising-Weihenstephan, Germany

$\uparrow$ Received 19 July 1996 mixing is considered poor, whereas if it is close to the mix composition, good mixing has been achieved. Because it is irrelevant whether the concentration of the sample deviates positively or negatively from the nominal concentration, the mean quadratic deviation is a good measure of mixing efficiency $[4,5]$. In statistics, this is called the square of the standard deviation $\sigma^{2}$. This is the theoretical variance of all possible samples in the mixer, and for a given mixture, $\sigma^{2}$ is a characteristic parameter that depends on the sample size. The mixing efficiency $\sigma^{2}$ therefore "improves" as the sample size increases [6]. If the sample size is of the same order as the particle size of at least one of the solids in the mixture, then $\sigma^{2}$ for the best case of mixing approaches a limiting value $\sigma_{z}^{2}$. This limiting value is a function of the sample collection conditions [6].

\section{Powder mixing mechanisms}

\subsection{The Fokker-Planck Equation}

Assuming that mixing arises from random motion of individual particles, Fokker and Planck developed the following differential equation which is based on the resulting random concentration changes that originate in the mixer:

$$
\frac{d c(x)}{d t}=-u \cdot \frac{d c(x)}{d x}+D \cdot \frac{d^{2} c(x)}{d x^{2}}
$$

This equation is identical in form to Fick's 2nd Law of Diffusion. It describes the concentration change at a given position in the mixer and includes two 
parameters: the transport coefficient $u$ and the dispersion coefficient $D . D$ is a measure of the random motion in the mixer and depends on the mobility of the individual particles. The transport coefficient $u$ corresponds to the convective flow present in the mixer. However, it is not set equal to the throughput of the mixer, and is thus also relevant for the case of batch mixing.

\subsection{Batch mixing}

According to Equation (1) there are two separate sources of mass flow: convective flow and dispersive (or diffusive) flow. In a batch mixer, these separate flows can work against each other, resulting in demixing [7]. Figure 1 shows the experiments carried out by Müller [7]. Demixing was brought about by the difference in transport characteristics between iron and quartz (straight line) and between iron and limestone (dotted line). In the first experiment, at the beginning the diffusion and transport streams were in the same direction and in the second, in the opposite direction. The empirical coefficients of variation reflect the course of events predicted in the model. Hence, the first experiment gave rise to a homogenous distribution and then to demixing, and a random homogeneous distribution was not attained at any time in the second experiment.

Demixing can also occur when no convective transport processes are present. This results because the dispersion coefficient is a function of concentration (or location). Because the dispersion coefficient is strongly coupled with the particle mobility, it has a longer residence time for the component in the zones with smaller dispersion coefficients. Statistically, this results in demixed zones within the mixer.

Without convective transport and assuming a constant dispersion coefficient $D$, the Fokker-Planck Equation reduces to:

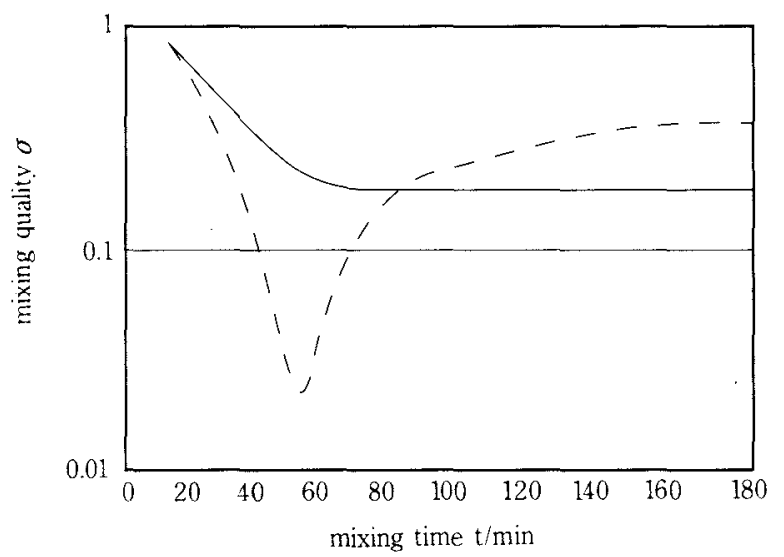

Fig. 1 Demixing tendency caused by convective flow in a batch mixer [7]

$$
\frac{d c(x)}{d t}=D \cdot \frac{d^{2} c(x)}{d x^{2}}
$$

This is the well-known form of Fick's 2nd Law of Diffusion. It can be written in dimensionless form with $\lambda=\mathrm{x} / L$ and $\tau=t / T$ as follows:

$$
\frac{d c(\lambda)}{d \tau}=+\frac{d^{2} c(\lambda)}{d \lambda^{2}}
$$

$T=L^{2} / D$ is a characteristic time formed from a characteristic mixer length $\mathrm{L}$ and the dispersion coefficient $D$. For the sample case of a closed barrel mixer of length $L$, the equation can be solved in closed form [7]. Rigid walls are assumed as boundary conditions, i.e. there is no material flow at $x=D$ and $x=L$ over the wall. The initial condition is that the component to be considered is highly concentrated on one side of the mixer.

If the variation coefficient $\sigma / \mu$, related to the mean value $\mu$, is used to represent the mixing efficiency, one obtains the following simple formula:

$$
\frac{\sigma}{\mu}=\sqrt{2} \cdot e^{-\left(\frac{\pi}{L}\right)^{2} \cdot D \cdot t}
$$

The equation predicts what is observed in practice: as the dispersion coefficient increases, mixing occurs more rapidly.

Experimental results from Müller [17] are depicted in double logarithmic form in Figure 2 as an example of dispersive mixing. The system consisted of 300-400 $\mu \mathrm{m}$ copper particles mixed with the same size nickel particles of concentration $c_{O}=7.67 \cdot 10^{-3}$. From the slope of the initial line, one obtains a dispersion coefficient of $D=1.1 \mathrm{~cm}^{2} / \mathrm{s}$. This coefficient is practically independent of the length/diameter ratio

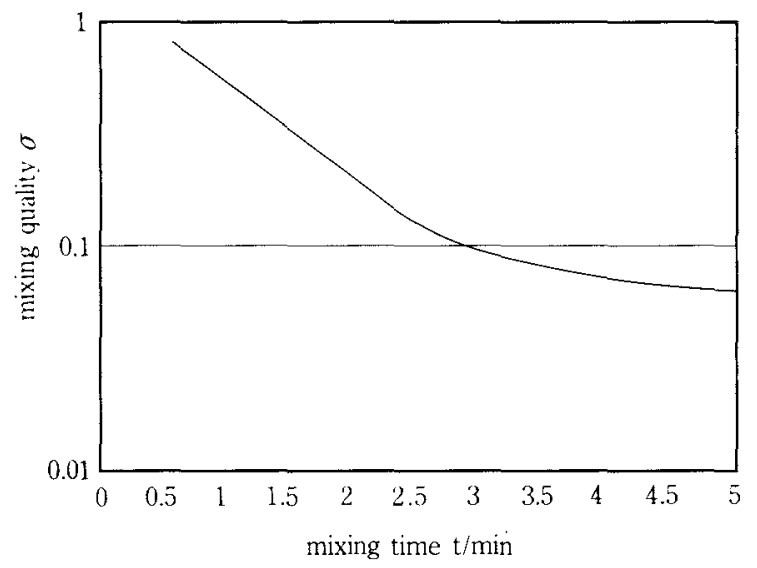

Fig. 2 Mixing quality as a function of time for purely dispersive mixing [7] 
of the mixer. After a sufficiently long mixing time, sample collection error becomes the dominant influential parameter and the measured points begin to asymptotically approach a constant value. The centre of gravity of one of the components was initially near the wall (due to the initial boundary conditions), and after the mix process, was exactly in the centre of the mixer, indicating that it is now homogeneously distributed. Thus, even without convective transport, the centre of gravity of a component shifts solely due to dispersive transport.

\section{Continuous mixing of the two material streams}

\subsection{Depiction of the mixing process}

At the inlet to an axial barrel mixer, two material streams converge into the mixer such that no backflow occurs. The material streams are mixed at the entrance and at each point in the mixer radially (i.e. ideal cross-flow mixing). Generally, a retaining weir is positioned at the end of the mixer and the excess material flows out over it. The degree of fill of both components at the weir is designated $\phi_{a}$. Due to the dispersive and/or convective transport processes, both of the material streams, which are homogenous in the radial direction, are conveyed to the opposite end of the mixer. As discussed in the introduction, one of the primary requirements of continuous mixing is to damp out concentration oscillations resulting from periodic injections of material.

Each of the material streams can be described by the Fokker-Planck Equation:

$$
\begin{aligned}
\frac{d \phi_{1}(\chi)}{d t} & =-u_{1} \cdot \frac{d \phi_{1}(x)}{d x}+D_{1} \cdot \frac{d^{2} \phi_{1}(x)}{d x^{2}} \\
\frac{d \phi_{2}(\chi)}{d t} & =-u_{2} \cdot \frac{d \phi_{2}(x)}{d x}+D_{2} \cdot \frac{d^{2} \phi_{2}(x)}{d x^{2}}
\end{aligned}
$$

Here, $\phi_{1}(x)$ and $\phi_{2}(x)$ correspond to the masses of components 1 and 2 , respectively, per differential mixer volume. They are therefore proportional to the local degree of fill of each component and are normalised by the reference degree of fill $\phi_{a}$ at the exit.

In general, both material streams have their own unique dispersive and convective transport coefficients. However, if both streams consist of nearly identical or very similar substances and differ, for instance, only in their colour, the coefficients for the two materials may be assumed to be equal (i.e. transport coefficient $u_{1}=u_{2}=u$, and the dispersion coefficient $D_{1}=D_{2}=D$ ).

As was seen in the batch case, the transport coefficient $u$ is a property of the mixer. In most investigations and literature reports, $u$ is set equal to the mean material transport. This assumption is, however, not valid in all cases. It may be approximately fulfilled when one of the material streams dominates, conveying the other stream without significantly dispersing it. Such a situation occurs frequently when the primary mass flow is a fluid that, upon entering the mixer, displaces a fluid that is already present in the mixer. However, in particular for the mixing of two powder streams, the above assumption is not valid, and therefore both FokkerPlanck equations must be considered.

\subsection{Boundary conditions for continuous mixing of two components}

The two mass streams to be mixed enter the mixer at one end $(x=0)$. At this end, no material is allowed to exit the mixer. Both or either of the components individually are subject to fluctuations.

The time-dependent deviation of the concentration from the mean is a measure of the heterogeneity of the mixture, and is expressed by the variance $\sigma_{0}^{2}$ or standard deviation $\sigma_{0}$, or by the variation coefficient $\sigma_{0} / \mu$.

The mixed material is discharged over the retaining weir. In the literature, a convective discharge $u \phi_{a}(L)$ is often postulated. However, once again, this boundary condition is only valid for the case in which one of the material streams dominates with purely convective flow.

The boundary conditions for the retaining weir are: if the transport (dispersive or convective) causes the degree of fill at the end of the mixer to rise above the given discharge height, the overflow (which has a concentration corresponding to this location and time in the mixer) is immediately discharged. The result is a constant local degree of fill at the end of the mixer, in accordance with requirements.

The discharged material is also subject to other more minor fluctuations. As in the case of the inlet flow, the heterogeneity of material exiting the mixer can be characterised by a variance or variation coefficient. Based on conservation of mass, at steady state the mean value of the output concentration must be equivalent to the mean value at the entrance $\mu$.

The goal of mixing is obviously to reduce theheterogeneity of the entrance material $\sigma_{0}$ to produce 
a quality product. The ratio $\sigma / \sigma_{0}$ is a measure of the effectiveness of the mixing process.

\section{Simulation of a continuous stochastic mix- ing process}

The simulation of continuous mixing using Equation (5) reveals an unexpectedly strong dependence on the dispersion coefficient (Figure 3).

It is well known in batch mixing that a larger dispersion coefficient leads to more rapid mixing (Equation 4). One expects the same to be true for continuous mixing. The dispersion coefficient is also associated with back-mixing, which is intuitively expected to be necessary for successful mixing. The simulation indicates that this expectation is not completely correct. Depending on the value of the transport rate $u$, the mixed material can be either accelerated or decelerated as the dispersion coefficient increases. An analysis of the mass distribution in the mixer shows that the magnitude of the dispersion coefficient has no influence when the convective transport in the mixer is adequate to convey the material. There is thus no variation in concentration or degree of fill within the mixer and thus no dispersive influence. At low transport rates, the degree of fill decreases to the end of the mixer and the dispersive effect supports the mass transport. With shorter residence times, the mixing results become poorer. For larger convective transport rates, the degree of fill increases up to the outlet and a larger degree of dispersion reduces the overall mixer throughput. In this case, the mixing efficiency is improved as the dispersion coefficient increases.

Simulations conducted by varying different parameters show that the most influential parameter is the residence time (related to the characteristic fluctuation time $T$ of the entrance material streams).

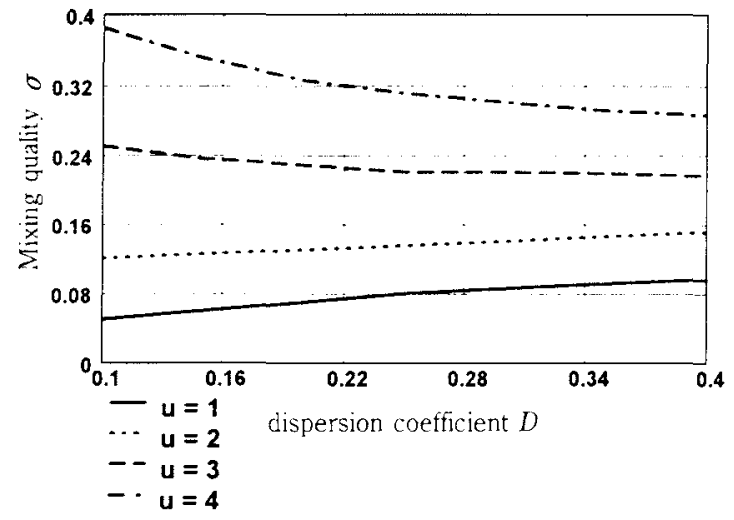

Fig. 3 Mixing quality as a function of dispersion coefficient $D$ and transport coefficient $u$
It also indicates that the mixing efficiency falls within a narrow band, which can be completely described by a mastercurve (Figure 4) [8].

Unfortunately, there are few accounts in the literature in which practical results have been analysed. Some recent results (shown in Figure 4) are available in the work by Weinekötter $[9,10]$. These experimental results show surprisingly good agreement with the master curve, and the difference can be explained based on the step oscillation applied at the entrance section, which causes better mix results for "high frequency" components.

\section{Silo mixing}

There is a wide variety of silo mixers that are frequently used for powder mixing. A common feature is that different material streams are mixed in the silo via external (or internal) blending and recirculation. The differences in the flow times within the mixing silo result in a range of residence times, i.e. materials introduced simultaneously at the inlet are conveyed to the outlet at different times, producing a mixture. The mixing effect is increased by circulating the contents several times within the silo. In contrast to a stochastic process, mixing in a silo mixer is very much a deterministic process. Schulze [11] developed a cell / layer model that simulates the processes in a mixer. His derivation results in a vector expression for the time-dependent concentration distribution $C_{k}$ in the mixer:

$$
C_{k+1}=M \cdot C_{k}
$$

Here, $M$ is the mixer matrix and contains the residence time spectrum data. The matrix can be used to assess the effectiveness of the silo mixing process.

Using a modification of the above equation, Dauth

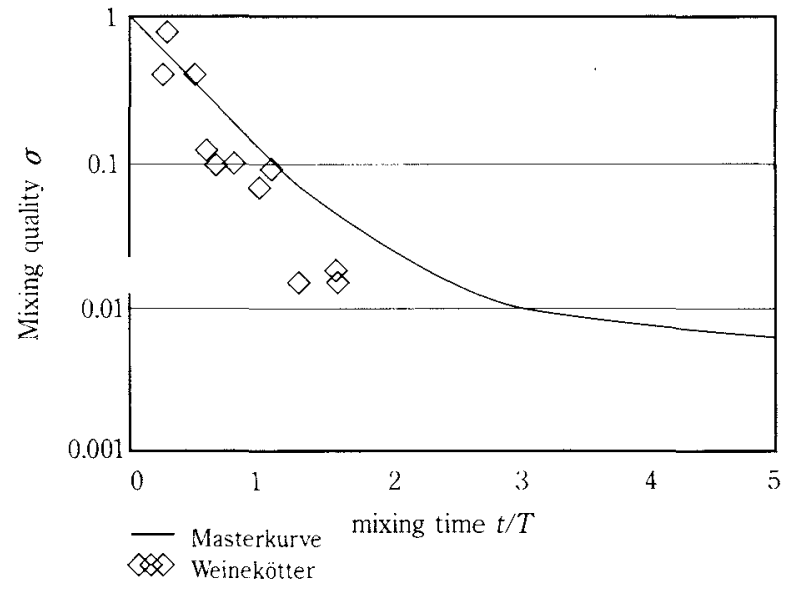

Fig. 4 Master curve for the mixing quality [8] 
and Sommer [12] simulated the concentration profile at the outlet of a silo mixer (Figure 5). They used a convolution method whereby the entrance conditions were continuously changed (due to recirculation). Analogous to the Schulze equation, the only input to the model is the residence time distribution of the product in the silo, and this can be easily determined via Dirac marking. Comparison of the simulation with mixing results in a plain silo showed excellent agreement through numerous recirculations. It will thus be possible to use simulation to optimise residence time spectra and thereby provide guidance on the proper design of silo mixers.

\section{Conclusions}

The theoretical variance $\sigma^{2}$ was defined and proposed as a characteristic parameter to measure the quality of mixers and mixed materials. The empirical variance $\sigma^{2}$ is a measure of mixing efficiency with an associated degree of uncertainty and within a given range (confidence interval).

The Fokker-Planck Equation is useful for describing the mixing process and simulating a stochastic mixer with two comparable material streams (in particular, for the blending of powders). The simulation method used in the literature exclusively up to now, in which the transport coefficient $u$ is determined from the throughput, is not generally valid. In addition, boundary conditions at the outlet must account for the practical case of overflow at the retaining weir.

The commonly held view that a large dispersion coefficient (corresponding to significant recirculation) improves mixing was found to be valid for batch mixing. However, in continuous mixing, the effect of the dispersion coefficient depends on the magnitude

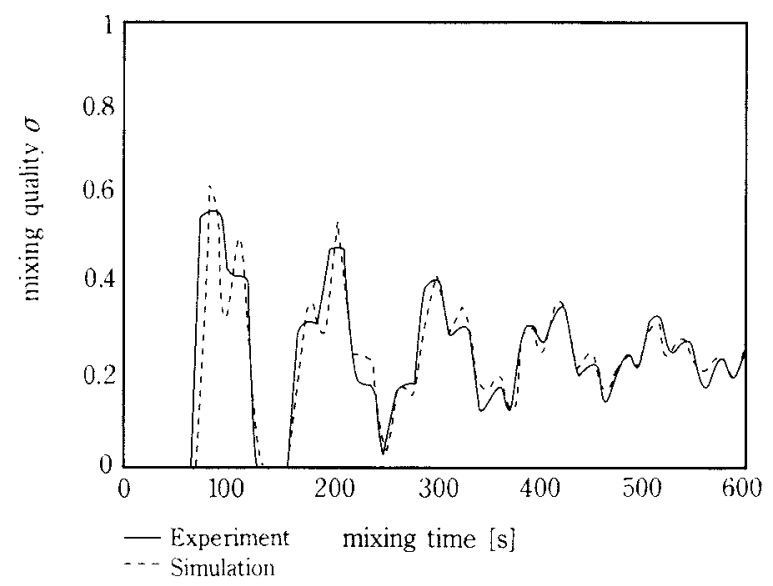

Fig. 5 Comparison between experiment and simulation of a silo mixer of the "convective" transport rate.

From the simulation, it appears that obtaining sufficiently accurate mix results for practical applications depends only on the relationship between the residence time and the fluctuation time of the entrance material streams. The experiments of Weinekötter confirm this result.

As is the case for stochastic mixers, modern simulation methods can also be used with deterministic silo mixers, both to predict mixing results and provide insight into their design. Such methods provide scientific tools for analysing powder mixing, which, unfortunately, has been almost exclusively an empirical field to date. These techniques thus offer great potential to further advances in this area.

\section{List of symbols:}

$C$ : concentration matrix

$c(x)$ : concentration at point $x$

$D$ : dispersion coefficient

$L \quad$ : length of mixer

$M$ : mix matrix

$t \quad:$ time

$T$ : characteristic time

$u \quad$ : transport coefficient

$x$ : coordinate

$\phi \quad$ : filling rate

$\lambda$ : dimensionless coordinate

$\mu \quad$ : concentration of the total mix

$\sigma_{2}:$ standard deviation

$\sigma^{2}$ : variance

$\sigma_{0}:$ standard deviation in the beginning

$\sigma_{z}:$ standard deviation of the random mix

$\tau$ : dimensionless time

\section{Literature}

[1] Harnby N., Edwards M.F., Nienow A.W.: Mixing in the Process Industries; Butterworths London 1985

[2] Sterbacek Z., Tausk P.: Mixing in the Chemical Industry; Pergamon Press Oxford 1965

[3] Koichi Inoya, Keishi Gotoh, Ko Higashitani: Powder Technology Handbook; Marcel Dekker, Inc. New York 1991

[4] Poole K.R., Taylor R.F.: Trans. Instn. Chem. Eng \$2 (1964) T166

[5] Williams J., Khan M.L.: The Chem. Eng. 269 (1973)19

[6] Sommer K.: Sampling of Powders and Bulk Materials; Springer-Verlag New York 1986

[7] Müller W.: Untersuchungen über Mischzeit, Mischgüte und Arbeitsbedarf in Mischtrommeln mit rotierenden Mischelementen; Dissertation Karlsruhe 1966

[8] Sommer K.: Powder Mixing, Preprints 1st Int. Particle Technology Forum Denver 1994 
49] Weinekötter R., Gericke H.: Mischen von Feststoffen; Springer Verlag Berlin 1995

[10] Weinekötter R.: Kontinuierliches Mischen feiner Feststoffe; Dissertation ETH Zürich 1993
[11] Schulze D.: Schüttgut 1(1995) 3 483/486; Schüttgut 1 (1995) 4 615/618; Schüttgut 2 (1996) 2 241/247

[12] Dauth H., Sommer K.: Silo Mixing; Preprints World Congress on Chemical Engineering San Diego; 1996

\section{Author's short biography}

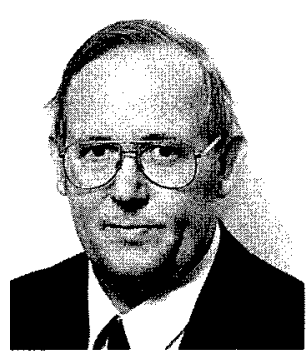

\section{Karl Sommer}

Karl Sommer, born in Ludwigshafen, Germany, on the 24th June 1943, studied Process Technology with the main point of emphasis on physical systems at the Technical University of Karlsruhe. Upon completion of his studies in 1968, he worked on the academic staff at the Institute for Mechanical Process Technology of the Technical University of Karlsruhe, simultaneously pursuing his doctorate, which he earned in 1974. Thereafter, he worked as scientific assistant at the Institute for Mechanical Process Technology for three years before taking the post of team manager at BASF in Ludwigshafen. In 1977, he qualified as a university lecturer for the branch of study "Mechanical process technology" at the Technical University of Karlsruhe. Since 1982, Karl Sommer has held the chair at the faculty of brewing, foodstuffs technology and dairy science at the Technical University in Munich.

He has also had several works published by Springer Publishers, among them "Probenahme von Pulvern und körnigen Massengütern"' in 1979, This work was translated into English and appeared as "Sampling of Powders and Bulk Materials" in 1986. 